\title{
INVESTIGATING DYSPEPSIA IN CLINICAL PRACTICE - A TRAP FOR GIARDIA
}

\author{
Banovcin P. Jr., Demeter M., Bozikova J., Hyrdel R.
}

Department of Internal Medicine - Gastroenterology, Jessenius Faculty of Medicine, Commenius University and University Hospital Martin, Slovak Republic

\begin{abstract}
Introduction: Dyspepsia is a very common condition with significant morbidity and economic implications. Dyspeptic symptoms have heterogeneous pathogenic mechanisms, including several organic, systemic or extragastrointestinal causes, however, origin of the most cases of dyspepsia remains unclear. This study aimed to focus on potential causes in patients with nonspecific dyspeptic symptoms in absence of organic, systemic or biochemical and hematological findings that readily explain their symptoms.

Methods: We studied presence of persistent abdominal symptoms (heartburn, dull epigastric pain, epigastric cramps, epigastric fullness, flatulence, diarrhoea), weight loss. All patients (116 patients, 29 males/87 females) had normal laboratory tests, negative abdominal ultrasound evaluation and upper endoscopy findings. Presence of Giardia intestinalis (GI), Helicobacter pylori (HP) infection and coeliac disease (CD) was evaluated by obtaining biopsy samples and duodenal fluid aspiration during upper endoscopy. We determined the effect of eradication treatment on dyspepsia symptoms 2 months after therapy.

Results: HP infection was present in $28 \%$, coeliac disease in $6 \%$, GI infection was diagnosed in $27 \%$ patients GI and HP in $12 \%, \mathrm{CD}$ and HP in $1 \%$, GI and CD in $2 \%$. None of these causes were presented in $39 \%$. Most frequent symptoms were epigastric fullness (69 \%), dull epigastric pain (38\%), heartburn (35\%), flatulence (34\%), abdominal cramps (31\%) and diarrhoea (16\%). We documented the remission of symptoms in $79 \%$ examined patients 2 month after eradication therapy.

Conclusion: The most common cause of dyspepsia was Helicobacter pylori infection and chronic giardiasis. Although H. pylori and coeliac disease are widely well known, we pointed out Giardia intestinalis as another possible cause of dyspepsia. Chronic giardiasis leads in selected individuals to dyspeptic symptomatology that mimics functional dyspepsia and irritable bowel syndrome. We recommend test for G. intestinalis infection in all patients with chronic dyspeptic complaints of unknown origin.
\end{abstract}

Key words: Dyspepsia, functional dyspepsia, Giardia intestinalis, Helicobacter pylori, coeliac disease

\section{INTRODUCTION}

Dyspepsia is a very common condition with significant morbidity and economic implications. Around $25-40 \%$ of adults in the general population have dyspepsia, but the prevalence and incidence depend on population study design, observation period, and definition of dyspepsia, itself $(1,2)$. According to the Czech study, the prevalence of long-lasting dyspeptic symptoms in uninvestigated dyspepsia was $17 \%$ (3). Dyspepsia accounts for 2-5\% of primary care consultations, but patients that are most affected often do not seek medical care (4). A large number of patients in clinical practice present symptoms such as epigastric pain, abdominal cramps, excessive belching, early satiation, postprandial fullness or nausea. The dyspeptic symptoms can have several organic, systemic or extragastrointestinal causes. However, the origin of most of the cases of dyspepsia remains unclear. When these symptoms are chronic and occur in the absence of organic disease that readily explains them, patients are considered to have a functional gastroduodenal disorder (5). Functional dyspepsia remains a diagnosis of exclusion. The therapeutical

Address for correspondence:

Banovcin P.Jr. MD, Clinic of Internal Medicine, Gastroenterology Dept., Jessenius Faculty of Medicine CU and University Hospital in Martin, Kollarova Str. N. 2, 03601 Martin, Slovak Republic

email: pbanovcin@gmail.com 
and diagnostical processes are often not optimal and still controversial. Due to these facts we focused on potential causes of non-specific dyspeptic symptomatology.

Giardia intestinalis (also known as Giardia lamblia and Giardia duodenalis) is an enteric protozoan pathogen found in a variety of mammalian hosts, including humans, and causes one of the most common parasitic infections worldwide. It contributes to estimate of 280 million symptomatic human infections per year (6). One of the major sources of infection in humans is contaminated water; however, there is evidence that the parasite is transmitted by human-to-human contact or by the contact with the domestic or wild animals (7). The symptoms of human giardiasis are highly variable. They vary from asymptomatic to severe form with diarrhoea, fever, weight loss and malabsorbtion. Most prominent clinical symptoms are abdominal pain, vague abdominal discomfort, bloating, flatulence and diarrhoea. Various extra-intestinal manifestations have been reported such as maculopapular rush, pulmonary infiltrates, polyarthritis and urticaria $(8,9)$. Chronic courses are common, but are mostly asymptomatic or presented by non-specific mild symptoms that may result in a low clinical index of suspicion for the diagnosis. Chronic infection may be linked to the phenomenon of antigenic variation and individual response of the host. Recent data show that Giardia intestinalis infection has been found to trigger abdominal symptoms of functional gastrointestinal disorder such as irritable bowel syndrome or functional dyspepsia. $(10,11,12)$

\section{MATERIAL AND METHODS}

We enrolled to our study 116 patients (29 males/87 females) aged 18 years and over attending Gastroenterology Outpatient Unit due to chronic dyspeptic symptoms. Patients with persistent abdominal dyspeptic symptoms in duration at least 3 months were considered for the study. Patient with the alarm features (such as dysphagia, odynophagia, vomiting, bleeding or rapid weight loss) as well pregnant women and patients familiarly for malignant disease were excluded. All patients underwent complex physical examination, routine biochemical and hematological blood laboratory tests.

Furthermore, ultrasound evaluation including hepatobiliary tract, pancreas, kidneys and upper endoscopy were performed. All examination had not produced relevant explanation

for dyspepsia. Upper gastrointestinal endoscopy was accompanied by obtaining of multiplied biopsy samples from gastric antrum to investigate presence of Helicobacter pylori (H. pylori) infection. Duodenal biopsy samples (D2-D3) and serum anti-gliadin, anti-endomysial and anti-transglutaminase antibodies estimation was used to evaluate coeliac disease. Presence of Giardia intestinalis infection was performed by direct examination of duodenal juice aspirate. Aspiration of duodenal fluid was performed on the fasting patient, during the endoscopy so the position of the aspiration tube was correctly placed in the distal duodenum or proximal jejunum. Aspirates were immediately transported in sterile containers in a tempered medium to microbiology laboratory. The samples were processed within 2 hours for microscopic examination.

We studied presence of $\mathrm{H}$. pylori infection, G. intestinalis and coeliac disease and its relative contribution to overall dyspeptic symptoms (heartburn, dull epigastric pain, epigastric cramps, epigastric fullness, flatulence, diarrhoea). After identification of possible cause, appropriate treatment was initiated (eradication of $\mathrm{H}$. pylori, gluten-free diet in coeliac disease and therapy with metronidazole $250 \mathrm{mg}$ tid. In the case of giadriasis, we determined the effect of eradication treatment on functional dyspepsia symptoms 2 months after therapy. Statistical analysis was performed using the program PAST version 1.80. 


\section{RESULTS}

One hundred and sixteen patients (87F, 29M) fulfilled the inclusion criteria. The mean age of patients was 37.3 (18-67years). Giardia intestinalis infection was diagnosed in 27 $\%$ of the patients. $\mathrm{H}$. pylori infection was found in $28 \%$, coeliac disease in $6 \%$. Both G. intestinalis and $\mathrm{H}$. pylori were present in $12 \%$, coeliac disease and $\mathrm{H}$. pylori in $1 \%$, giardiasis and coeliac disease in $2 \%$. There were $39 \%$ of the patients that did not exemplify any of the causes mentioned. Figure 1. Most frequent symptoms were epigastric fullness (69 \%), dull epigastric pain (38\%), heartburn (35\%), flatulence (34\%), abdominal cramps (31\%), and diarrhoea (16\%) Figure 2. Comparing frequency of symptoms, we didn't confirmed statistically significant differences among investigated groups. Average lenght of dyspeptic syndrome history was 18.5 months and average weight loss was $2 \mathrm{~kg}$.

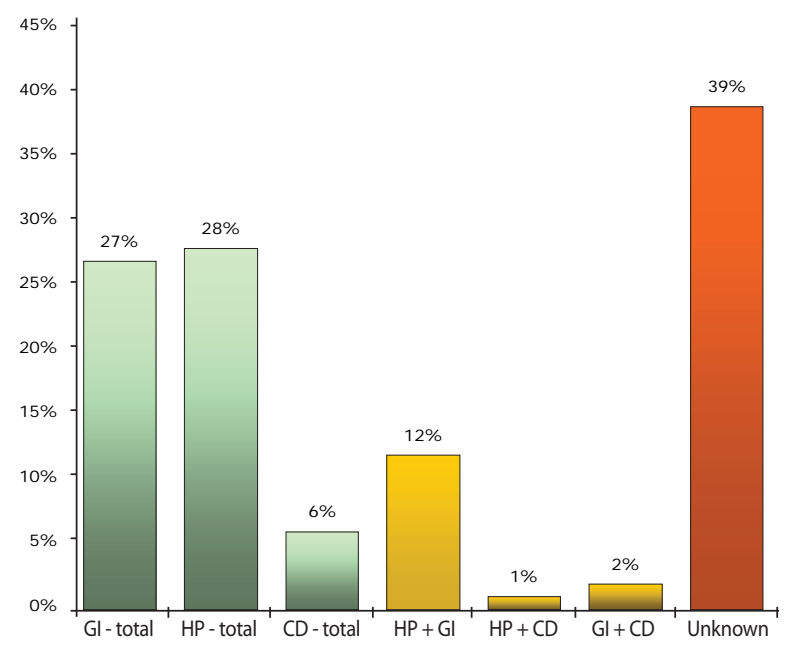

Fig. 1. Summary of potential dyspepsia causes in study patients (totally). Giardia intestinalis (GI), Helicobacter pylori (HP) and coeliac disease (CD).

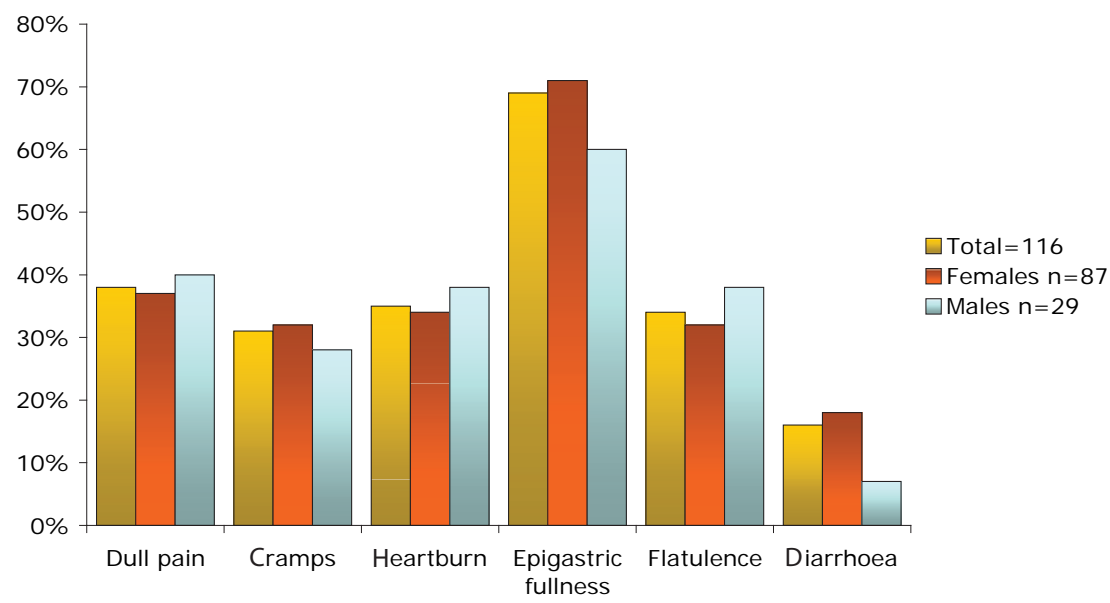

Fig. 2. Prevalence of selected dyspepsia symptoms in study patients (totally) 
In the Giardia positive group was the most frequent symptom was epigastric fullness (86\%), dull epigastric pain (50\%). Frequencies of other symptoms are shown in Figure 3. Average length of dyspeptic syndrome was 23.3 months in total G. intestinalis infection (32 months in isolated infection) with weight loss of $2.8 \mathrm{~kg}$ (3.5 giardia isolated). Fourteen patients with giardiasis (43\%) had simultaneous $\mathrm{H}$. pylori infection. We documented the remission or significant improvement of symptoms in $79 \%$ of Giardia positive patients 2 month after therapy (treatment with metronidazole). Approx. $7 \%$ of patients did not response to therapy, and $14 \%$ of the patients cancelled their follow up, thus we do not have any response for that group. Figure 4.

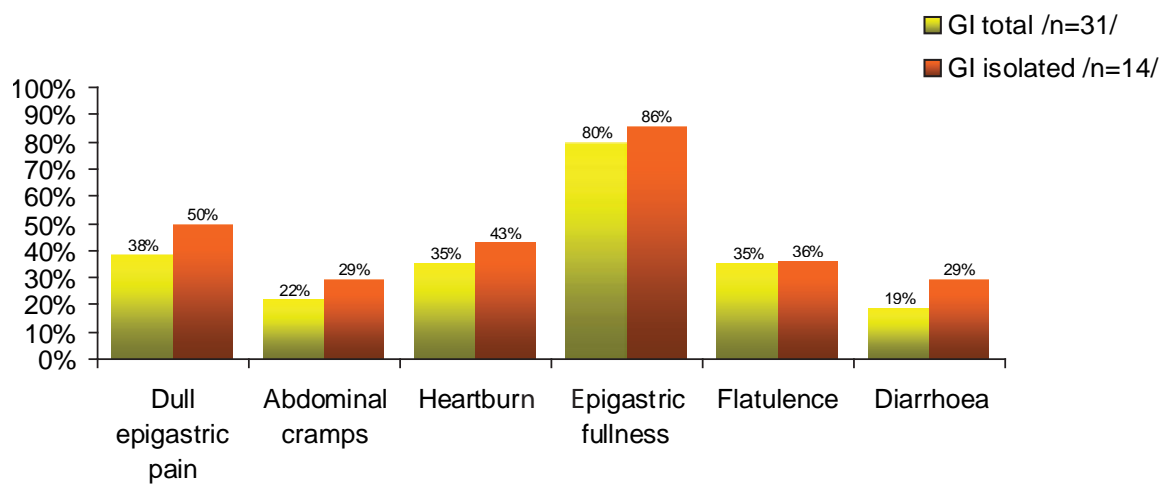

Fig. 3. Prevalence of symptoms in Giardia intestinalis positive patients.

Persistence of symptoms after therapy in patienst with GI

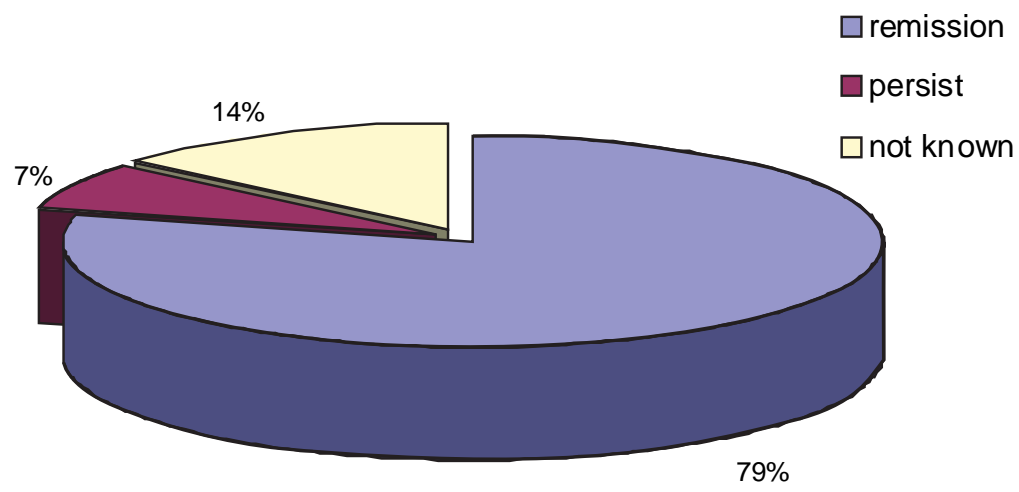

Fig. 4 Effect of 14 days metronidasole 250mg tid. treatment on dyspepsia symptoms. Evaluated 2 months after treatment. 


\section{DISCUSSION}

Dyspepsia is common and global problem with board differential. This study aimed to focus on patients with nonspecific dyspeptic symptoms in absence of organic, systemic or biochemical and hematological findings that explain their symptoms. In order to prevent serious illness, patient complaining of alarm symptoms were excluded. The negative aspect of this study was our failure to fulfill the diagnosis of functional gastrointestinal disorders according to ROME III criteria. However, the aim of the study was to identify other possible agents that may contribute to development of gastrointestinal symptoms. The role of $\mathrm{H}$. pylori in functional disease has been controversial. Recent meta-analyses suggest some benefit from $\mathrm{H}$. pylori eradication (13). In our findings was the $\mathrm{H}$. pylori infection most common agent. After eradication therapy, $72 \%$ of patients reported benefits from this therapy.

Coeliac disease was diagnosed in $6 \%$. As expected, flatulence fullness and diarrhoea were the dominant symptoms, but not significantly different from other groups. On the other hand, in our study were not sufficiently many patients with coeliac disease to conclude any decision.

Incidence of giardiasis varies from $20 \%$ to $60 \%$ in endemic areas and $2-7 \%$ in industrialized countries depending on region and age (6). We found out that $27 \%$ of studied patients had giardia infection. The rate is higher compared to general population in western countries mostly due to selection of symptomatic patients.

Traditional diagnosis of giardiasis is set by the direct detection of trophozoites or cysts in a stool sample; however, repeated stool examinations are needed to avoid false-negative results, because cyst excretion may be intermittent. This may be difficult sometime in outpatient units because of patient compliance. The data show that histological examination of duodenal biopsies is unsuitable $(15,16,17)$. In recent years, rapid diagnostic tests that use antigen detection methods or PCR have been employed. These tests are both highly specific and sensitive, but still expensive (14). The specificity and sensitivity is also questionable in case of chronic giardiasis. Diagnosis of Giardia intestinalis in our study was performed by microscopic evaluation of duodenal juice aspirate. There are studies that show yields of microscopic examination of duodenal aspirate that vary from $0.7 \%$ to $47.6 \%(15,16)$. There is disadvantage to duodenal fluid aspiration in that it is performed during endoscopy therefore it is not suitable for routine screening. Another disadvantage of this evaluation is that aspirate should be preceded within 2 hours of aspiration and transported in temperate medium.

Giardia intestinalis infection in humans has a wide spectrum of clinical manifestation ranging from asymptomatic to serious diarrhoea. In our study the most frequent symptom was abdominal fullness and diarrhoea followed by heartburn, dull epigastric pain abdominal cramps, and flatulency. There were no symptoms or symptom-complex that reliably allowed the recognition of giardiasis. Again our results were similar to previously published studies. $(17,18)$

The pathophysiology of these symptoms in giardia infection has not been well understood. The possible mechanisms include direct damage to the intestinal brush and mucosa resulting in secondary lactose intolerance, reduced intestinal absorption and deconjugation of bile salts. Recent hypothesis also suggests induction of apoptosis in intestinal epithelial cells and disruption of tight junctional zona-occludens followed by increased epithelial permeability across epithelial monolayers. $(19,20,23)$ Compared to other studies, diarrhoea was not so common feature in our study. as reported from in endemic areas. This difference might be caused by low initial dose of giardia cysts, in non-endemic area therefore the host is able to maintain parasite count at very low level, or it may be due to the distribution of different pathogenic strains. But this assumption is highly speculative. The association with clinical manifestation and parasite genotype is not definitive to date $(18,21,22)$. 
In our study, the mean age of patients with giardiasis was slightly lower (not significantly) compared to patients with $\mathrm{H}$. pylori infection and in the group without any findings. It is well know, that giardiasis is more common in children and adolescents (28). From this point of view, age may have some attribution in diagnostic process in uninvestigated dyspepsia and related symptoms.

Duration of symptoms was longest in patients with giardiasis. Possible reason of delayed diagnosis is that the G.intestinalis is generally considered to be on low degree of suspicion in non-endemic countries in comparison with the well-known H. pylori.

Importantly, we documented total remission of symptoms in $79 \%$ examined patients with G. intestinalis infection after 14-days course treatment with metronidazole at $250 \mathrm{mg}$ tid. There were no statistically significant differences in symptoms distribution or metronidazole therapy effect on them in all $\mathrm{G}$. intestinalis positive patients.

Another finding was that giardiasis was associated with $\mathrm{H}$. pylori infection. H. pylori was found in 14 patients of the 31 patients with giardiasis. This possibly reflects the fact that both $\mathrm{G}$. intestinalis and $\mathrm{H}$. pylori share similar risk factors (24). There is existing literature and data supporting possible synergy effect of infections, however, in our study were no significant differences between the giardia groups in symptoms presentation or distribution. Some authors suggest possible synergistic participation leading to metronidasole resistance and gastrointestinal neoplasia $(24,25,26,27)$.

Causal treatment of dyspesia led to resolution of dyspeptic symptoms; however in almost 39 \% patients we did not find the cause of dyspepsia. Such patients are hypothesized to have functional gastrointestinal disorder. Given that functional gastrointestinal disorders are the diagnosis per exlusionem, further examinations are needed to proof the diagnosis according to ROME III criteria.

\section{CONCLUSION}

Chronic dyspeptic syndrome is a frequent problem of patients visiting gastroenterology outpatient unit. In our study most common cause of dyspepsia were Helicobacter pylori infection, chronic giardiasis and coeliac disease. Although $\mathrm{H}$. pylori and coeliac disease are widely well known, we pointed out another possible cause of dyspepsia. According to our data, we are suggesting that chronic Giardia intestinalis infection leads to nonspecific dyspeptic symptomatology in selected individuals that mimics functional dyspepsia and irritable bowel syndrome. We recommend test GI infection in all patients with chronic dyspeptic complaints of unknown origin,

\section{REFERENCES}

1. Talley NY, Vakil NB, Moayyedi P. American Gastroenterological Association technical review on the evaluation of dyspepsia. Gastroenterology2005; 129:1756-80.

2. McQuaid KR. Dyspepsia. In: Feldman M, Friedmann LS, Brandt LJ, editors. Sleisenger \& Fordtran's gastrointestinal and liver disease. 8th ed. Philadelphia: Saunders Elsevier; pp. 2006;121-142.

3. Rejchrt S, Koupil I, Kopacova M, Vorisek V, Seifert B, Pozler O, Zivny P, Douda T, Palicka V, Holcik J, Bures J, European society for Primary care Gastroenterology. Prevalence and sociodemografic determinants of uninvestigated dyspepsia in the Czech republic.; Eur J gastroenterol Hepatol. 2008;Sep;20(9):898-905

4. Baron JH, Sonnenberg A. Hospital admissions and primary care attendances for non ulcer dyspepsia, reflux oesophagitis and peptic ulcer in Scotland 1981-2004. Eur J Gastroenterol Hepatol 2008;20:180-6.

5. TackJ, Talley NJ, Camilleri M et al. Functional gastroduodenal disorders. Gastroenterology 2006; 130:14661479.

6. WHO Protozoan Parasites (Cryptosporidium, Giardia, Cyclospora). Microbiological Agents in Drinking Water, 2nd edition. Addendum; 2002.

7. Hunter PR, Thompson RC. The zoonotic transmission of Giardia and Cryptosporidium.Int J Parasitol. 2005; Oct;35(11-12):1181-90. 
8. M. Letts, D. Davidson and F. Lalonde, Synovitis secondary to giardiasis in children, Am J Orthop 1998;27 pp. $451-454$

9. Nenoff P, Domula E, Willing U, Herrmann J.Giardia lamblia-cause of urticaria and pruritus or accidental association? Hautarzt. 2006;Jun;57(6):518-20, 521-2.

10. Hanevik K, Dizdar V, Langeland N, Hausken T. Development of functional gastrointestinal disorders after Giardia lamblia infection. BMC Gastroenterol.2009; 9: 27.

11. D’Anchino M, Orlando D, De Feudis L. Giardia lamblia infections become clinically evident by eliciting symptoms of irritable bowel syndrome. J Infect 2002; 45:169-172.

12. Grazioli B, Matera G, Laratta C, Schipani G, Guarnieri G, Spiniello E, et al. Giardia lamblia infection in patients with irritable bowel syndrome and dyspepsia: a prospective study. World J Gastro-enterol 2006; 12:1941-1944.

13. Moayyedi P, Soo S, Deeks J, Delaney B, Harris A, Innes M, Oakes R, Wilson S, Roalfe A, Bennett C, Forman D. Eradication of Helicobacter pylori for non-ulcer dyspepsia. Cochrane Database Syst Rev. 2006 Apr 19;(2):CD002096.

14. Garcia LS, Shimizu RY. Evaluation of nine immunoassay kits (enzyme immunoassay and direct fluorescence) for detection of Giardia lamblia and Cryptosporidium parvum in human fecal specimens. J Clin Microbiol 1997; 35:1526-1529

15. Diagnostic yield of duodenal aspirate for G. lamblia and comparison to duodenal mucosal biopsies.Gupta SK, Croffie JM, Pfefferkorn MD, Fitzgerald JF. Dig Dis Sci. 2003 Mar;48(3):605-7.

16. McHenry R, Bartlett MS, Lehman GA, O'Connor KW: The yield of routine duodenal aspiration for Giardia lamblia during esophagogastroduodenoscopy. Gastrointest Endosc 1987; 6:425-426.

17. Yakoob J, Jafri W, Abid S, Jafri N, Hamid S, Shah HA, Rizvi L, Islam M, Shaikh H. Giardiasis in patients with dyspeptic symptoms. World J Gastroenterol. 2005;Nov 14;11(42):6667-70

18. Read CM, Walters J, Robertson ID, Thompson RCA Correlation between genotype of Giardia duodenalis and diarrhoea. Int J Parasitol 2002; 32:229-231

19. Chin AC, Teoh DA, Scott KG, et al. Strain-dependent induction of enterocyte apoptosis by Giardia lamblia disrupts epithelial barrier function in a caspase-3-dependent manner. Infect Immun 2002; 70:3673-3680

20. Scott KG, Meddings JB, Kirk DR, et al. Intestinal infection with Giardia spp. reduces epithelial barrier function in a myosin light chain kinase-dependent fashion. Gastroenterology 2002; 123:1179-1190

21. Homan WL, Mank TG. Human giardiasis. genotype linked differences in clinical symptomatology. Int $\mathrm{J}$ Parasitol 2001; 31:822-826

22. Sahagun J, Clavel A, Goni P, Seral C, Llorente MT, Castillo FJ, Capilla S, Arias A, Gomez-Lus R. Correlation between the presence of symptoms and the Giardia duodenalis genotype. Eur J Clin Microbiol Infect Dis 2008; 27(1):81-83

23. Das S, Schteingart CD, Hofmann AF, Reiner DS, Aley SB, Gillin FD. Giardia lamblia: evidence for carriermediated uptake and release of conjugated bile acids..Exp Parasitol. 1997; Oct;87(2):133-41.

24. Moreira ED Jr, Nassri VB, Santos RS, Matos JF, de Carvalho WA, Silvani CS, Santana CS. Association of Helicobacter pylori infection and giardiasis: results from a study of surrogate markers for fecal exposure among children. World J Gastroenterol 2005; 11: 2759-2763

25. Abou Holw SA, Anwar MM, Heshmat MG, Enany AY, Rashad MM. Effect of concommitant Helicobacter pylori infection in patients with Giardia lamblia in Egypt. J Egypt Soc Parasitol. 2009; Aug;39(2):439-46.

26. Land KM, Johnson PJ. Molecular basis of metronidazole resistance in pathogenic bacteria and protozoa. Drug Resist Update 1999; 2: 289-294

27. Patterson MM, Schrenzel MD, Feng Y, Fox JG. Gastritis and intestinal metaplasia in Syrian hamsters infected with Helicobacter aurati and two other microaerobes. Vet Pathol 2000; 37: 589-596

28. Farthing MJ. Giardiasis. Gastroenterol Clin North Am 1996; 25: 493-515

Received: December,1,2010

Accepted: January,6,2011 